\title{
Design and Optimisation of Composite Corrugated Skin for a Span Morphing Wing
}

\author{
Yuying $\mathrm{Xia}^{1}$ \\ University of the West of Scotland, Paisley PA1 2BE, UK \\ Rafic M. Ajaj ${ }^{2}$ \\ University of Southampton, Southampton SO17 1BJ, UK \\ and \\ Michael I. Friswell ${ }^{3}$ \\ Swansea University, Swansea SA2 8PP, UK
}

\begin{abstract}
The concept of span morphing aircraft is attracting a lot of attention due to the demands to enhance flight performance and control authority. Corrugated laminates provide a good solution for the skin of a span morphing wing due to their extremely anisotropic behaviour. Although corrugated skins have been proposed for camber morphing, they have not been proposed for span morphing. Hence the main focus of this paper is to optimise the geometry of the corrugated skin to minimize the axial stiffness, with constraints of material strength limits and out-of-plane deformations due to the aerodynamics. Two aerodyanic solvers, Tornado Vortex Lattice Method (VLM) and XFOIL, are compared and a Genetic Algorithm (GA) is used as the optimizer. Parametric modeling and calculation by ANSYS APDL was used to generate the finite element model and perform the analysis automatically based on the global and local geometric parameters. The parameter sensitivity is also analyzed and discussed. The optimization results show that XFOIL is more accurate than VLM for this analysis. The maximum displacement constraint causes the optimizer to choose fewer corrugations rather than increase the sheet thickness. This implies that the thickness has a higher impact than the number of corrugations on the axial stiffness. The optimization results show that the corrugation height and angle are maximized as they seem to have less influence on the maximum out-of-plane displacement.
\end{abstract}

\section{Nomenclature}

$=$ chord length

$=$ length between wing sections

$=$ number of corrugations between wing sections

$=$ half height of corrugaton

$=$ trapezoidal corrugation angle

$=$ thickness of original sheet of the corrugated skin

$=$ longitudinal Young's modulus of the original sheet

$=$ transverse Young's modulus of the original sheet

$=$ longitudinal tensile strength of the original sheet

$=$ transverse tensile strength of the original sheet

$=$ longitudinal compressive strength of the original sheet

$=$ transverse compressive strength of the original sheet

$=$ Poisson's ratio of the original sheet

\footnotetext{
${ }^{1}$ Lecturer, School of Engineering, y.xia@uws.ac.uk, AIAA Senior Member.

${ }^{2}$ Lecturer, Aeronautics and Astronautics,r.ajaj@southampton.ac.uk.

${ }^{3}$ Professor, College of Engineering, m.i.friswell@swansea.ac.uk.
} 


\section{Introduction}

$\mathrm{C}$ ontinuous demands to enhance flight performance and control authority have focused the interest of aircraft designers on span morphing. Wings with large spans have good range and fuel efficiency, but lack manoeuvrability and have relatively low cruise speeds. In contrast, aircraft with low aspect ratio wings can fly faster and are more manoeuvrable, but show poor aerodynamic efficiency [1]. A variable span wing can potentially integrate into a single aircraft the advantages of both designs, making this emerging technology especially attractive for military UAVs. Increasing the wingspan, increases the aspect ratio and wing area, and decreases the spanwise lift distribution for the same lift. Thus, the drag of the wing could be decreased, and consequently, the range or endurance of the vehicle increased. Unfortunately, the wing-root bending moment can increase considerably due to the larger span. Thus the aerodynamic, structural, aeroelastic, and control characteristics of the vehicle should be investigated in the design of variable-span morphing wings. Most span morphing concepts are based on a telescopic mechanism, following the ideas of Ivan Makhonine, a Russian expatriate, where the wing outer panel telescoped inside the inner panel to enable span and wing area changes. The MAK-10 was the first design with a telescopic wing and it first flew in 1931. The mechanism was powered pneumatically and enabled span increases up to $62 \%$ (from 13 to $21 \mathrm{~m}$ ) and area increases up to $57 \%$ (from 21 to $33 \mathrm{~m}^{2}$ ) [2]. With telescopic mechanisms there are two options. The first option is to telescope the wing sections into each other which is very efficient from a structural point of view as it allows each wing section to have a semi-monocoque construction. However, the discrete changes in the chord along the span due to the different widths of the telescopic sections are associated with an aerodynamic penalty. The second option is to use telescopic spars that are covered with some flexible skin. This is less efficient from a structural point of view especially since the flexible skin can only support a small part of the aerodynamic loads and the spars have to carry almost all of the loads. However, this option allows a uniform chord along the span which is superior from an aerodynamic point of view. The focus here is on the second option and to establish feasibility and potential benefits. Composite corrugated skins are a promising candidate to be integrated with the spars to allow flexibility in the spanwise/morphing direction while maintaining sufficient stiffness in the other directions to maintain the aerodynamic profile of the wing independent of morphing.

From a structural perspective, corrugated laminates [3] offer a good solution for the skin of a span morphing wing due to their extremely anisotropic behaviour: compliance in the corrugation direction that allows shape changes and increases in surface area; stiffness in the direction transverse to the corrugation which enables the aerodynamic and inertial loads to be carried. Currently the design of the morphing wing and the specification of the skin are performed independently. This approach is adequate for simple wing geometries (such as morphing trailing edges), but inadequate for more complex three-dimensional geometries (such as the morphing winglet [4]).

From the aerodynamic point of view, corrugated laminates have a significant disadvantage for chord morphing due to their poor aerodynamic performance. Whitehead et al. [5] and Thill et al. [6] performed wind-tunnel tests and CFD analysis of airfoils with corrugated skins in the aft one third of the chordwise section at Reynolds numbers between 250,000 and 1,000,000. Xia et al. [7] performed a similar analysis for the airfoil fully covered by corrugated skins.

However corrugated laminates may be a good solution for span morphing as the corrugation is transverse to the flow direction. For wings with a large aspect ratio the aerodynamics is approximately 2-D, and the flow is therefore predominantly along the corrugations. Of course there will be small pressure differences between the peaks and troughs of the corrugations, leading to a 3D flow, although the aerodynamic performance should be reasonable.

In this paper, the problem definition is summarized in Section II, followed by a description of the design suite, which consists of a Genetic Algorithm (GA) optimiser, aerodynamic load prediction and a parametric structural model. Then a parameter sensitivity study is performed and finally the results and conclusions are given in Sections $\mathrm{V}$ and VI.

\section{The problem definition}

The main focus in this paper is to optimise the geometry of the corrugated skin to minimize the axial stiffness. To simplify the analysis, only the root partition of the wing semi-span is modeled and optimised. Minimising the axial stiffness is the key objective function and driver of this study. A low axial stiffness allows a small actuation force and lower power requirements that results in a smaller and lighter actuation system. The global bending stiffness is also investigated to quantify the ability of the corrugated skin to carry the global bending loads, although the telescopic spars are not considered in this analysis and would carry the majority of these loads. The local out-of- 
plane deformations due to the aerodynamics should be limited to maintain the aerodynamic profile of the wing. It is also essential for the structural weight of the skin to be as low as possible to retain the benefits of the span morphing technology. In fact, the shape of the corrugated skin can have a significant impact on the nature of the airflow around the wing, unless coating or foam filling is used to cover the corrugations. Modeling the detailed 3D airflow would require a high fidelity aero-structural analysis, and this is the next stage of work that will be addressed by the authors. In this paper, a design suite is developed to model and optimise the skin.

\section{The Design Suite}

The suite consists of a low fidelity aerodynamic model coupled to a high-fidelity structural model integrated with a Genetic Algorithm optimiser to determine the best geometric parameters of the corrugation that gives the maximum ratio of bending to axial stiffness.

\section{A. Genetic Algorithm (GA) Optimiser}

Genetic Algorithms (GAs) are stochastic global search and optimisation methods. GAs mimic the metaphor of natural evolution by applying the principle of the survival of the fittest to produce successively better approximations to a solution. They follow a population-based approach which allows the optimisation process to be parallelized and hence to reduce the computational time. GAs start with an initial population consisting of various individuals, and each individual represents a particular solution to the problem. The population evolves over generations to produce better solutions. The "Matlab GA Toolbox", developed by Chipperfield et al. [8], is incorporated in this analysis. A fitness value is assigned to every individual of the initial population through an objective function that assesses the performance of the individual in the problem domain. Then, individuals are selected based on their fitness index and crossover between them is performed to generate new offspring. Finally, mutation of the new offspring is performed to ensure that the probability of searching any subspace of the problem is never zero. These above mentioned processes iterate until the optimum solution is achieved depending on the convergence criteria of the problem.

\section{B. Aerodynamics}

Two aerodynamic solvers are used in this study. The first solver is the Tornado Vortex Lattice Method (VLM) [9]. Tornado is a linear aerodynamics code, and thus discounts wing thickness and viscous effects. These limitations imply that Tornado can only be used for angles of attack up to $8-10^{\circ}$ for slender wings. The aerodynamic loads are extracted from Tornado and fed into the structure model. The mesh used is sufficiently fine to have a good representation of the pressure distribution over the wing.

XFOIL is an interactive program for the design and analysis of subsonic isolated airfoils, which was first developed by Mark Drela at MIT as a design tool for the MIT Daedalus project in the 1980s and further developed in collaboration with Harold Youngren. XFOIL combines the speed and accuracy of high-order panel methods with the new fully-coupled viscous/inviscid interaction method which used in the ISES code developed by Drela and Giles. XFOIL can calculate the pressure distribution on the airfoil and hence lift and drag characteristics by given the coordinates specifying the shape of a 2D airfoil, the Reynolds number and the Mach number.

\section{Parametric Modeling and the Calculation Module (PMCM)}

For optimization, the parametric modeling module is implemented within ANSYS APDL. The 3-D corrugated wing is generated automatically from the geometric parameters and the weight of the structure is calculated. In this analysis only two wing sections and the skin between them have been considered. Three cases with different boundary conditions are used to calculate the span extension stiffness, the span bending stiffness and the out of plane deformation under aerodynamic load. The maximum stress and maximum deflection are also obtained and form the constraints within the optimization.

\section{1) Parametric modeling}

The input parameters to generate the 3-D model of the corrugated wing are summarized in Table 1 . Note that all of the length parameters (except the thickness of the original sheet of the corrugated skin) are scale factors, which are equal to the original length divided by the chord length (SF). In this analysis a trapezoidal corrugation shape is used for the morphing skin. SHELL181 elements have been used to model the corrugated skin. 
Table 1 Input parameters for the 3-D corrugated wing model

\begin{tabular}{|l|c|l|}
\hline \multirow{4}{*}{ Global parameters } & Parameter & Description \\
\cline { 2 - 3 } & $\mathrm{SF}$ & Chord length, $c$ \\
\cline { 2 - 3 } & $\mathrm{AFM}$ & $\begin{array}{l}\text { Airfoil geometric matrix which include the } \\
\text { coordinate data of the airfoil (chord equal to 1) }\end{array}$ \\
\cline { 2 - 3 } & $\mathrm{LF}$ & Length factor between wing sections, $L / \mathrm{SF}$ \\
\hline \multirow{4}{*}{$\begin{array}{l}\text { Corrugation } \\
\text { geometric } \\
\text { parameters }\end{array}$} & $\mathrm{N}$ & Number of corrugations between wing sections \\
\cline { 2 - 3 } $\begin{array}{l}\text { Material } \\
\text { properties }\end{array}$ & $\mathrm{alpha}$ & Talf height of corrugation factor, $f$ /SF \\
\cline { 2 - 3 } & $\mathrm{t}$ & Orapezoidal corrugation angle, $\alpha$ \\
\cline { 2 - 3 } & $\mathrm{EX}$ & Young's Modulus, $E_{11}$ \\
\cline { 2 - 3 } & $\mathrm{EY}$ & Young's Modulus, $E_{22}$ \\
\cline { 2 - 3 } & $\mathrm{NU}$ & Poisson Ratio, $v$ \\
\hline
\end{tabular}

\section{2) Boundary Conditions}

The inner wing section is assumed to be rigid and is modeled using Multi-Point Constraints (MPCs) in ANSYS.

\section{Case 1: Axial Stiffness}

Case 1 focuses on pure extension in the span direction. The inner wing section (i.e. the inner rib) is clamped, and the outer wing section (i.e. outer rib) is displaced only in the span direction by the maximum span deformation $\Delta L$ required by the design. The reaction forces in the spanwise direction are then calculated and the span extension stiffness is estimated. The maximum stress within the corrugated skin is also estimated as a constraint within the optimization.

Case 2: Bending Stiffness

Case 2 focuses on pure global spanwise bending. The inner wing section (i.e. the inner rib) is clamped, and the outer wing section (i.e. outer rib) is displaced in the vertical direction by one unit. By comparing the reaction forces to those of a uniform beam an equivalent span bending stiffness is estimated.

Case 3: Aerodynamic Pressure Load

Case 3 investigates the effect of aerodynamic loads. The inner wing section (i.e. the inner rib) is clamped, and the outer wing section (i.e. outer rib) is simply supported. The boundary conditions represent the presence of a spar that carries the majority of the bending loads and the effect of the corrugation geometry on aerodynamics is not considered in this study. Two aerodynamic solvers are used in this case. In the Tornado VLM-based simulation, the aerodynamic pressure is extracted from Tornado VLM and applied at the lower surface of the corrugation. In the XFOIL-based investigation, the 2-D pressure distribution, which is extracted from XFOIL, is applied to both the upper and lower surfaces of the corrugated skin. We assume that the pressure distribution is uniform in the span direction, so that the 2-D aerodynamics from XFOIL is expanded to act on the 3-D corrugated skin.

\section{Parameter Sensitivity Study}

The work in this paper is based on the Tekever AR4 UAV. The UAV has a rectangular, unswept and untwisted wing. The design parameters and flight conditions used in this analysis are listed in Table 2.

Table 2 UAV Design Parameters.

\begin{tabular}{cc}
\hline \hline Parameters & Values \\
\hline Span & $1.8 \mathrm{~m}$ \\
Chord & $0.24 \mathrm{~m}$ \\
MTOW & $5 \mathrm{~kg}$ \\
Airfoil & NACA 0012 \\
Airspeed & $15.3 \mathrm{~m} / \mathrm{s}$ \\
AOA & $10^{\circ}$ \\
Altitude & $300 \mathrm{~m}$ \\
\hline
\end{tabular}


The parameters in Table are based on a 1-g level flight of the UAV. The Angle of Attack (AOA) is relatively high mainly because the airfoil used is uncambered. However, Tornado and XFOIL still work with good accuracy for an AOA of $10^{\circ}$.

\section{A. PMCM Tests}

Figure 1 shows the geometric model, which is generated by the parametric modeling techniques mentioned in Section III. The corresponding FEM mesh is shown in Figure 2. Other parameters, which have been defined in Section III, are: length between wing sections, $L=0.24 \mathrm{~m}$, the number of corrugations between wing sections, $N=5$, the half height of the corrugaton factor, $f / c=0.01, \alpha=\pi / 4$, and $t=5 \mathrm{~mm}$.
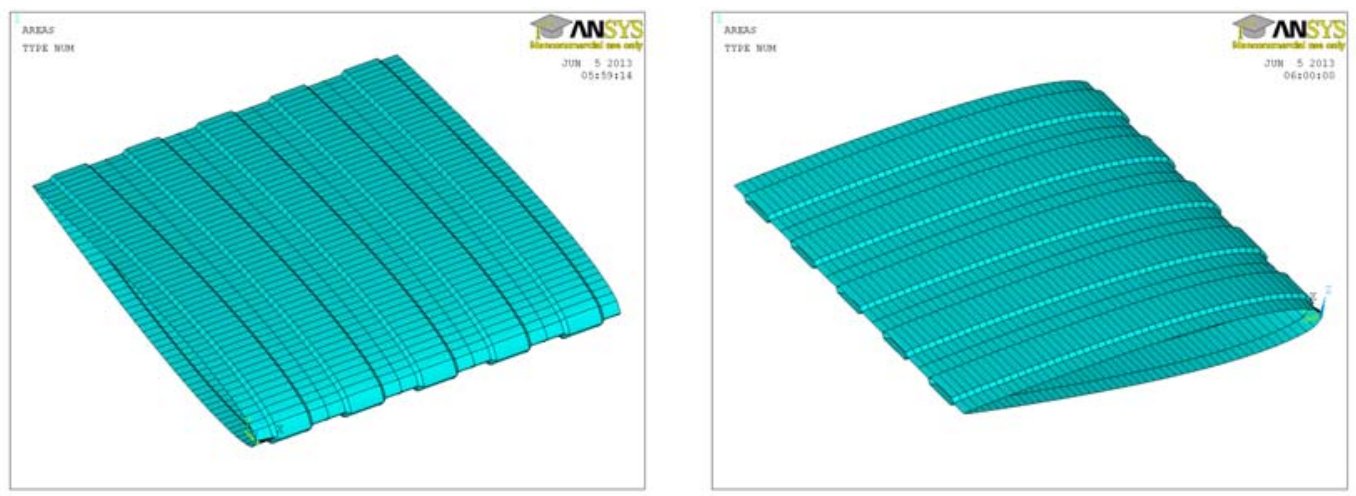

Figure 1 Parametrically generated geometric model

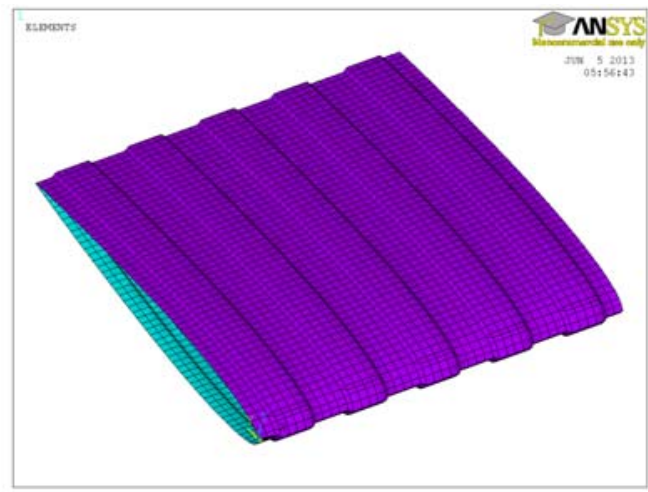

Figure 2 Parametrically generated FEM mesh

Figures 3 and 4 show the deformation shape and contours under pure extension (Case 1) and pure bending (Case 2), respectively, when the unit displacement applied for boundary conditions. Here, the material properties which have been defined in Section III are: $E_{11}=E_{22}=54 \mathrm{GPa}, v=0.26, \rho=1750 \mathrm{~kg} / \mathrm{m}^{3}$. The following strength limits are assumed: $X_{t}=Y_{t}=1780 \mathrm{MPa}$ and $X_{c}=Y_{c}=-700 \mathrm{MPa}$. 


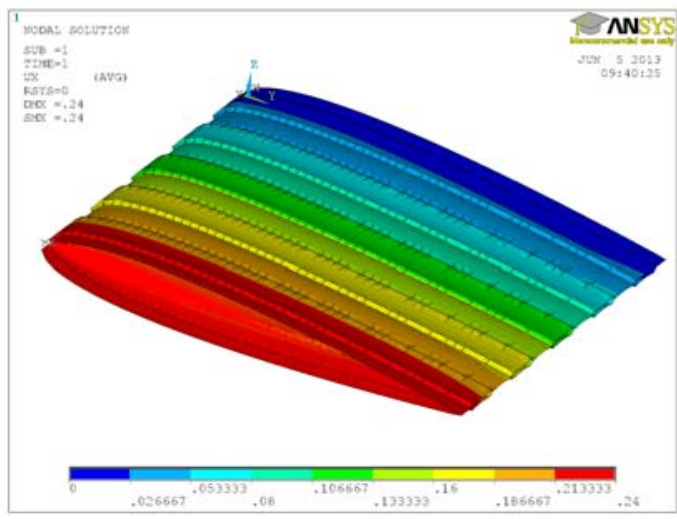

Figure 3 Span extension (Case 1)

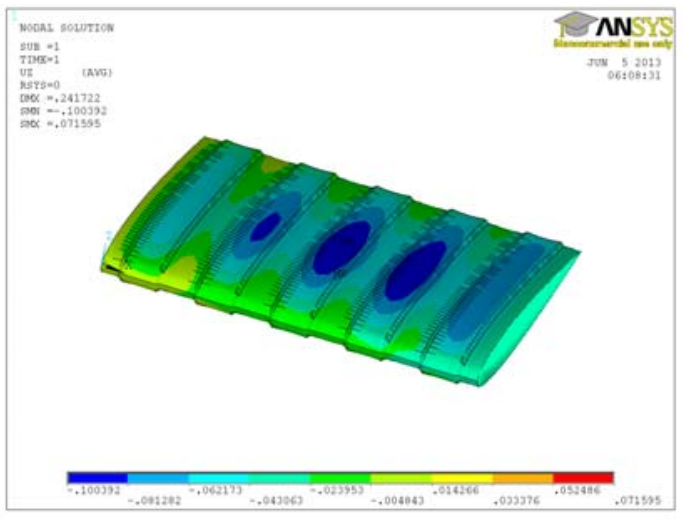

Figure 4 Spanwise bending (Case 2)

\section{B. Aerodynamic effects}

In the aerodynamic effects study we used the thickness of the original sheet as $t=0.5 \mathrm{~mm}$. Figures 5 and 6 indicate the aerodynamic load distributions which are extracted from Tornado VLM and XFOIL, respectively. Figure 5 shows the concentrated loads applied on the lower or upper surfaces of the corrugated skin and Figure 6 shows the 2-D pressure factor distribution of any wing section in the model (as mentioned before, the aerodynamics are assumed to be uniform in the spanwise direction). Figure 7 (a)-(c) shows the deflection contours due to the aerodynamics based on the Tornado VLM method with aerodynamic loads on the lower surface, the Tornado VLM method with aerodynamic loads on the upper surface, and XFOIL, respectively.

Figure 8 compares the deflections due to the aerodynamics based on the Tornado VLM and XFOIL methods, where the deformation of the wing section is predicted at $x=0.12 \mathrm{~m}$, which is the mid-section between the inner and out wing sections and where the maximum deflection should happen. The results show that the 3D corrugated wing is quite stiff and the maximum deflection is less than $0.02 \%$ of chord length in this case. The deflections are only slightly different when the aerodynamic load is applied on the upper or the lower surface using the Tornado VLM method. Hence, the concentrated loads will be applied on the lower surface of the corrugated skin in the following studies. The maximum deflection obtained using XFOIL is smaller than that obtained using the Tornado method, which indicates that the Tornado method will offer more conservative results during the optimization.

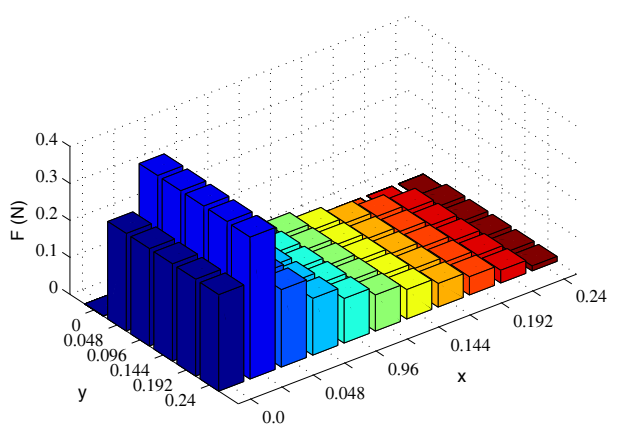

Figure 5Aerodynamic distribution (Tomado VLM)

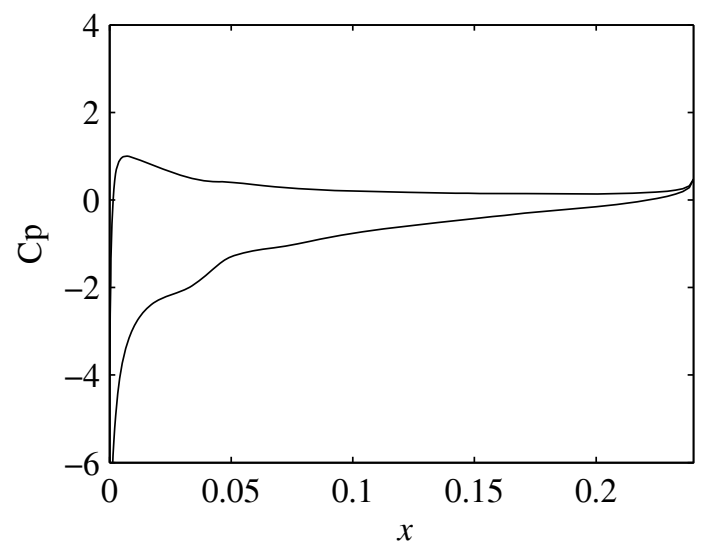

Figure 6 Aerodynamic distribution (XFOIL) 


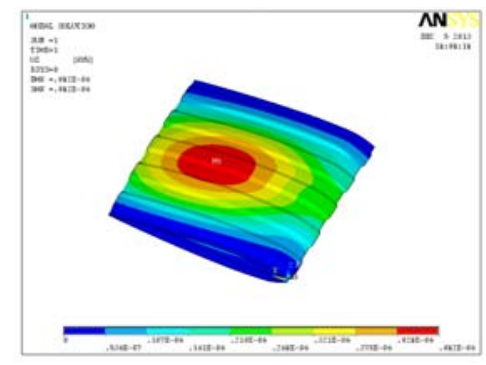

(a) VLM (loads on the lower suface)

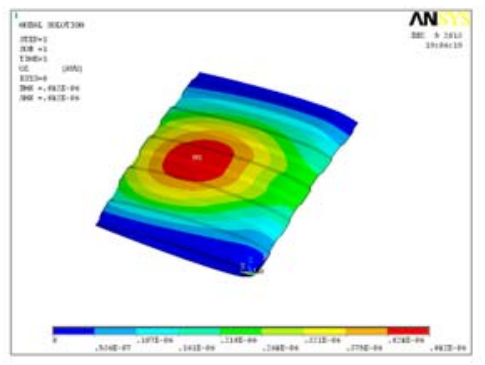

(b) VLM (loads on the upper suface)

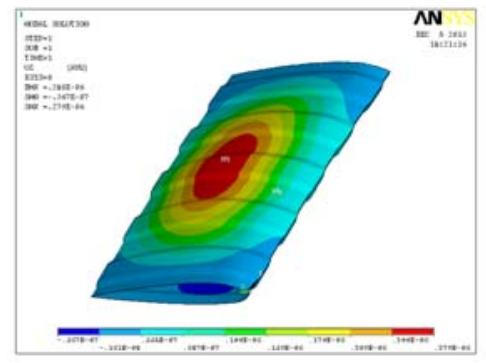

(c) XFOIL

Figure 7 Deflection contours due to the aerodyanamic pressure loads
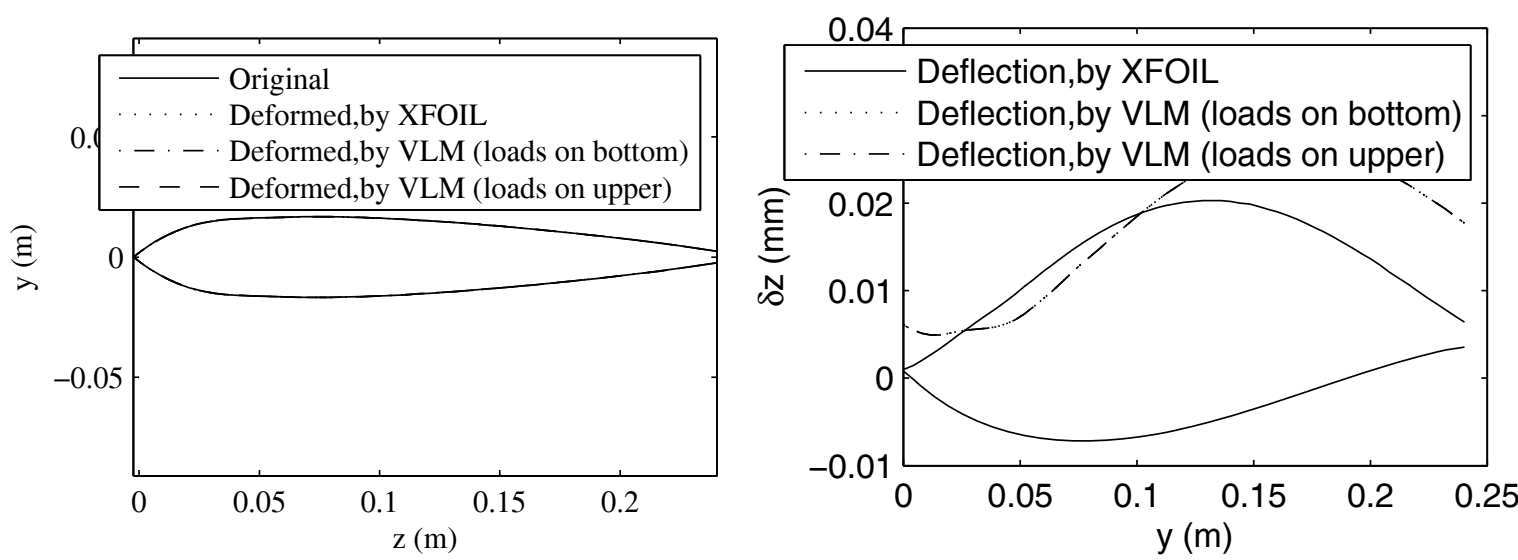

Figure 8 Deflection due to the aerodynamics (at $x=0.12 \mathrm{~m}$ )

\section{Parametric Analysis}

In this case, the corrugated skin is made of a glass fiber epoxy composite panel. The material properties are: Young's modulus $E_{11}=E_{22}=54 \mathrm{GPa}$, shear modulus $G_{12}=4.46 \mathrm{GPa}$, Poisson's ratio $v=0.26$. The thickness of the original sheet is $5 \mathrm{~mm}$. The NACA0012 airfoil has been used for the wing section. The chord length (outer corrugation) is $0.24 \mathrm{~m}$ and the length between two wing sections is $0.24 \mathrm{~m}$.

The axial stiffness varies with the number of corrugations, $N$, the trapezoidal corrugation angle, $\alpha$, and the half height of the corrugation, $f$, as shown in Figures 9 to 11, respectively. The variations of the global bending stiffness wwith these parameters are shown in Figures 12 to 14, and the variation of the total mass are shown in Figures 15 to 17.

The parametric sensitivity results show that the axial stiffness decreases as the number of corrugations, $N$, increases, as the trapezoidal corrugation angle, $\alpha$, increases and as the half height of the corrugation, $f$, increases. In contrast, the bending stiffness and the mass is less sensitive to these parameters. 


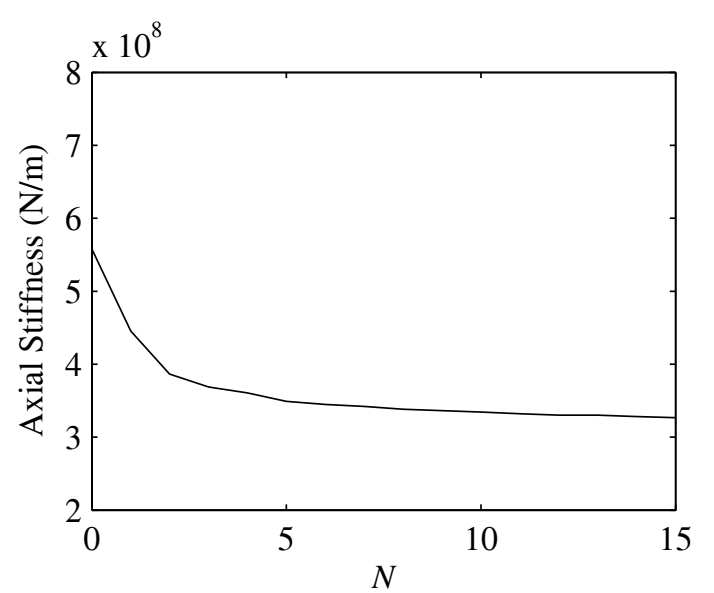

Figure 9 Variation of axial stiffness with $N$

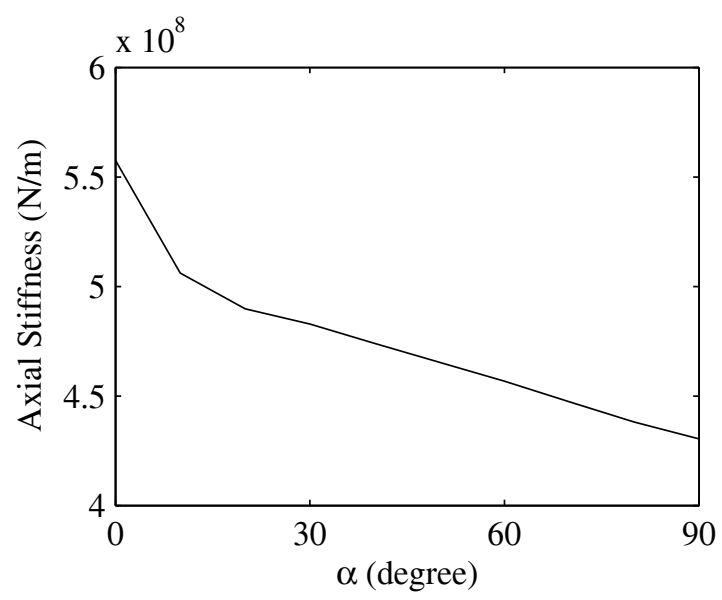

Figure 11 Variation of axial stiffness with $\alpha$

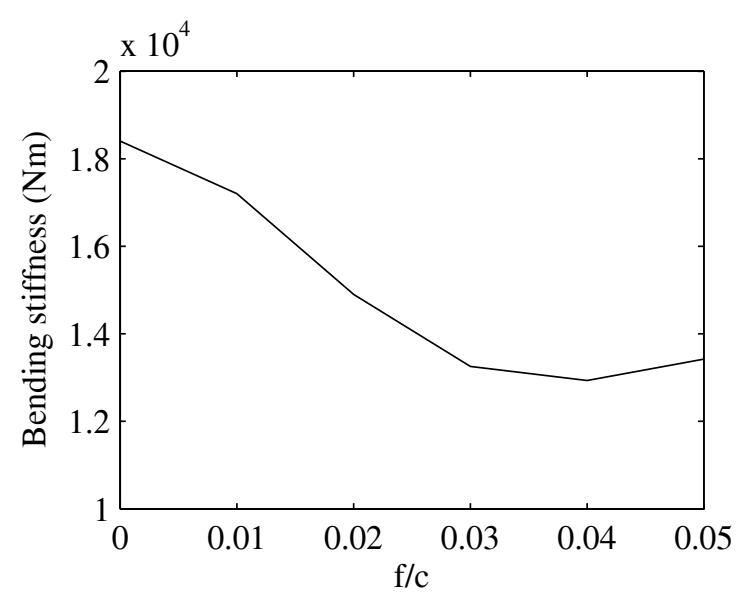

Figure 13 Variation of bending stiffness with $f / c$

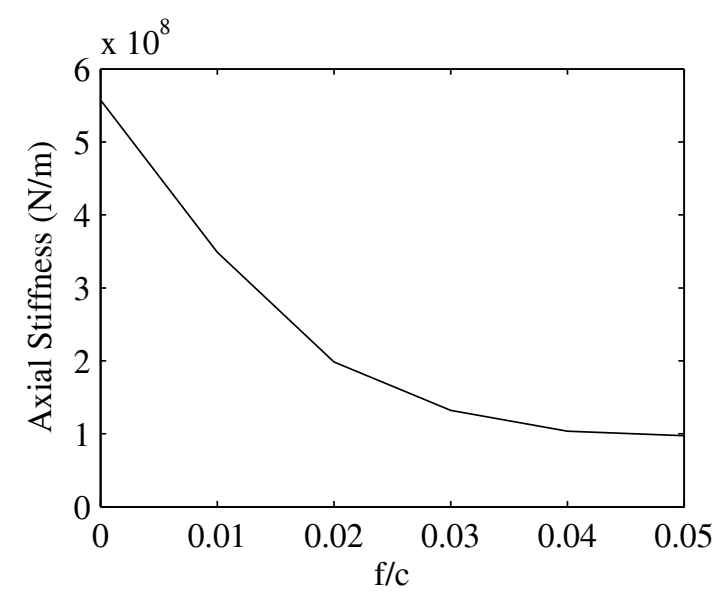

Figure 10 Variation of axial stiffness with $f / c$

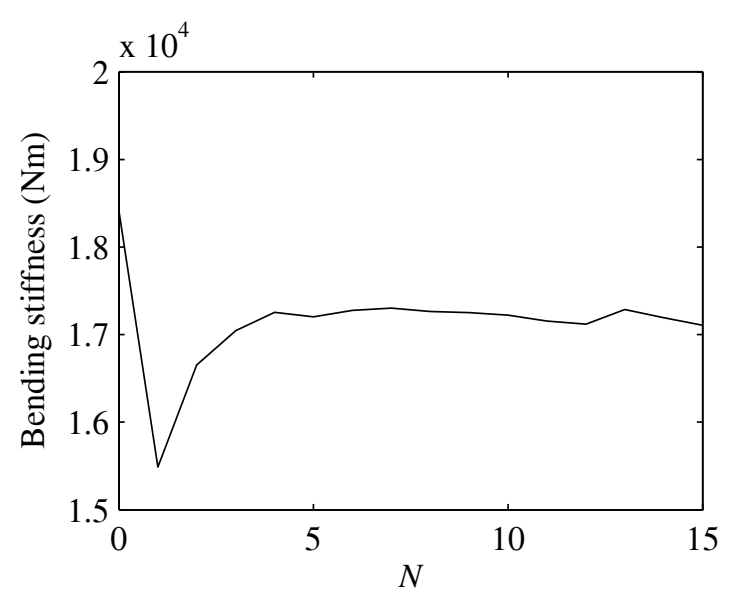

Figure 12 Variation of bending stiffness with $N$

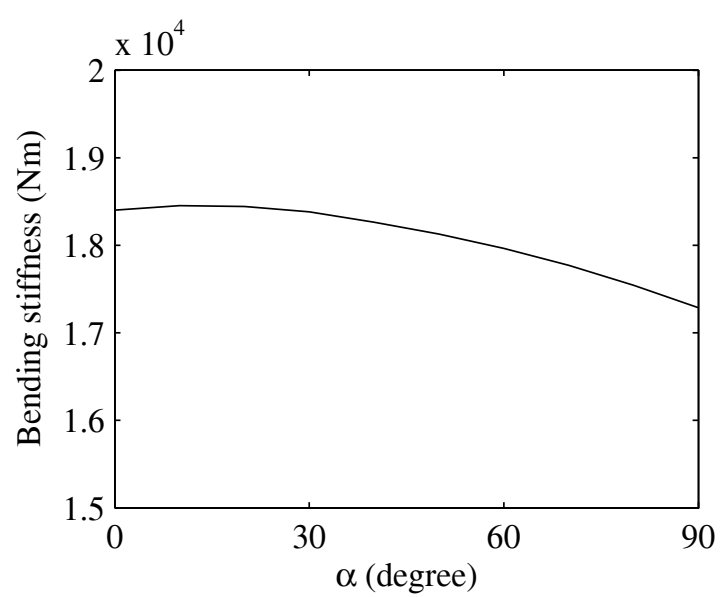

Figure 14 Variation of bending stiffness with $\alpha$ 


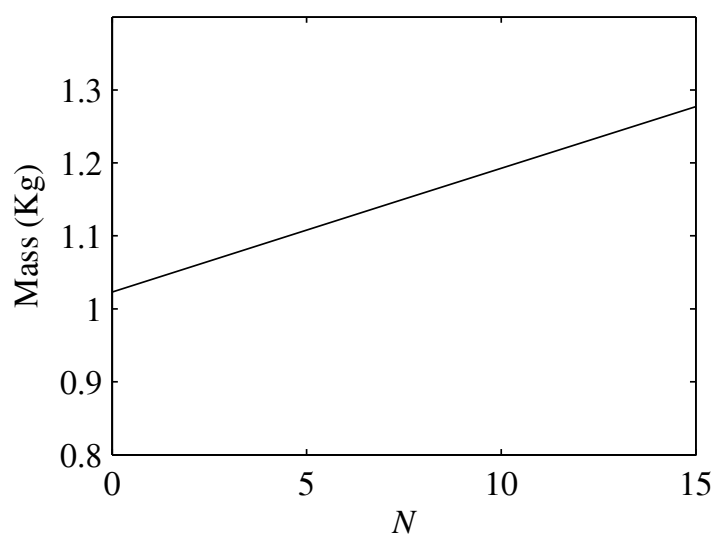

Figure 15 Variation of mass with $N$

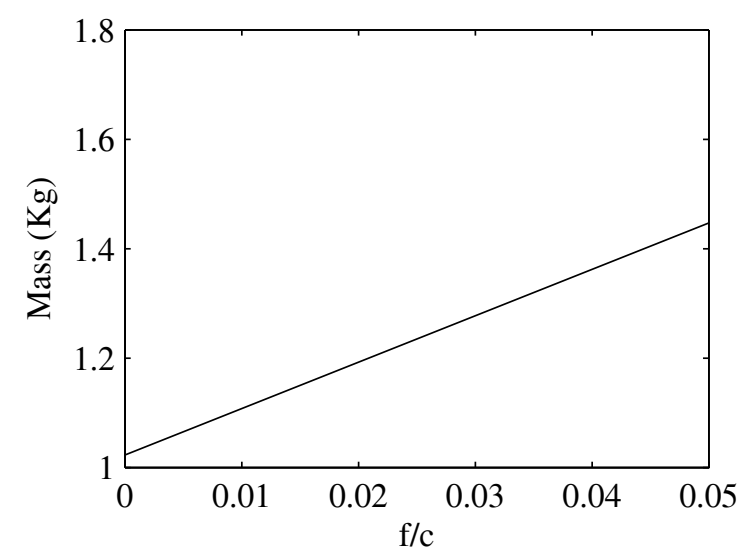

Figure 16 Variation of mass with $f / c$

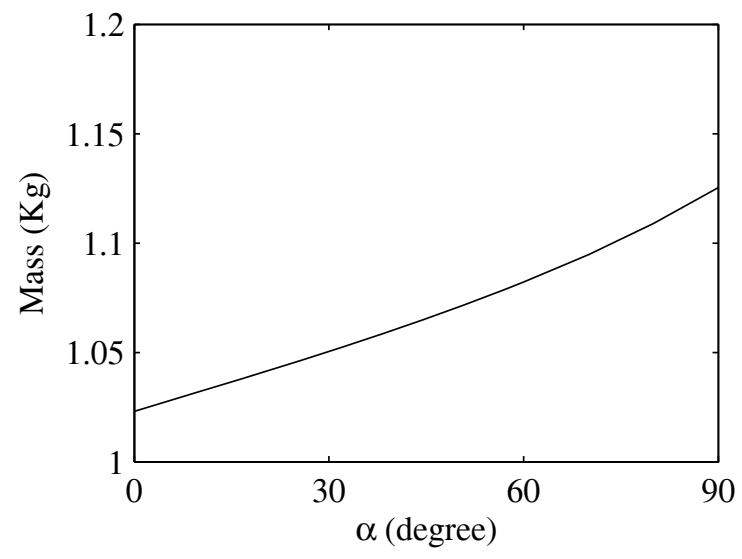

Figure 17 Variation of mass with $\alpha$

\section{Optimization Results}

The objective function is to minimize the axial stiffness of the sheet to be able to achieve an extension of $25 \%$. There are different ways to determine the convergence of the GA, and a series of convergence studies were performed. These showed that fixing the number of generations to 50 and the number of individuals to 50 is sufficient to yield robust results. It should be highlighted that in the optimization study the stresses induced by the $25 \%$ strain in the corrugation sheet are not considered. This will under-estimate the skin thickness required to withstand the aerodynamic and actuation loads. These issues will be considered in future work.

\section{A. With Tornado VLM}

Similar to the parametric study, the corrugation sheet used in this study starts from the wing root and has a span of $0.24 \mathrm{~m}$. The aerodynamic loads are extracted from Tornado for the flight conditions listed in Table 2 . These loads are in the form nodal forces that are applied on the lower side of the corrugation sheet. The optimization problem is summarized in Table 3. 
Table 3 Optimisation problem.

\begin{tabular}{cc}
\hline \hline Objective function & minimize $\left(K_{x}\right)$ \\
\hline Variables & $N, f, \alpha, t$ \\
\hline & $5 \leq N \leq 15$ \\
Constraints & $0.01 c \leq f \leq 0.1 c$ \\
& $10^{\circ} \leq \alpha \leq 90^{\circ}$ \\
& $0.1 \mathrm{~mm} \leq t \leq 10 \mathrm{~mm}$ \\
& $\delta_{\max } \leq 1 \mathrm{~mm}$ \\
\hline
\end{tabular}

To minimize the axial stiffness, the optimizer maximizes the number of corrugations, the height of the corrugations and the corrugation angle to the maximum allowable values listed in Table 3 . The maximum displacement of the lower side of the airfoil is much less than $1 \mathrm{~mm}$. The minimum axial stiffness of the sheet $\left(K_{x}\right)$ is $212 \mathrm{~N} / \mathrm{m}$.

Since the maximum displacement constraint is not violated, the span of the corrugation sheet is increased to 0.9 $\mathrm{m}$ while the chord and the depth are kept fixed as shown in Figure 18. This implies that the skin is covering the entire semi-span of the wing while supported at the wing root and tip only.

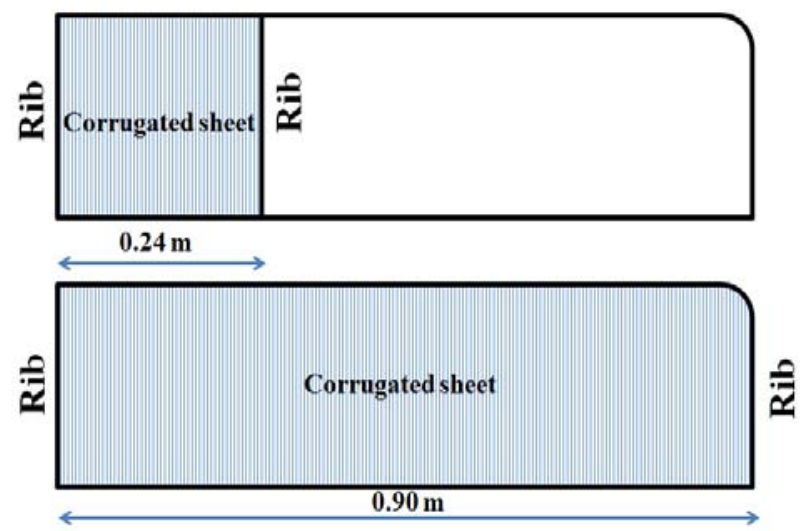

Figure 18 Geometry of the corrugation sheet over the wing semi-span.

The same optimization problem is repeated, with the only change that $N$ is now varied between 5 and 25 corrugations. The GA maximizes the corrugations height and the corrugation angle to the maximum allowable values. However, the optimum number of corrugations is 17 as increasing the number of corrugations further will violate the maximum displacement constraint. The minimum axial stiffness $\left(K_{x}\right)$ is $157 \mathrm{~N} / \mathrm{m}$. The results for each case are summarized in Table 4.

Table 4 Optimisation results with Tornado VLM.

\begin{tabular}{ccc}
\hline \hline Parameters & Span of $\mathbf{0 . 2 4} \mathbf{~ m}$ & Span of 0.9 m \\
\hline$N$ & 14 & 17 \\
$f$ & $0.0991 \mathrm{c}$ & $0.1 \mathrm{c}$ \\
$t(\mathrm{~mm})$ & 0.1 & 0.1 \\
$\alpha$ & $90^{\circ}$ & $90^{\circ}$ \\
$K_{x}(\mathrm{~N} / \mathrm{m})$ & 212 & 157 \\
$K_{z}(\mathrm{~N} / \mathrm{m})$ & 21 & 1.20 \\
\hline
\end{tabular}

\section{B. With XFOIL}

To obtain a better representation of the upper and lower deformations of the corrugation sheet, XFOIL is used to extract the 2D aerodynamic loads and then extrapolated along the length of the sheet to obtain the 3D loads. The design suite is modular and hence it is relatively simple to switch off Tornado and switch on XFOIL. 
The corrugation sheets (span of $0.24 \mathrm{~m}$ and $0.9 \mathrm{~m}$ ) are optimized again using the aerodynamic loads from XFOIL. Unlike the optimization in Section 3.1.1, the maximum deformations allowed are $1 \mathrm{~mm}$ for the $0.24 \mathrm{~m} \mathrm{span}$ and $5 \mathrm{~mm}$ for the $0.9 \mathrm{~m}$ span. The optimization results are summarized in Table 5.

Table 5 Optimisation results with XFOIL.

\begin{tabular}{ccc}
\hline \hline Parameters & Span of 0.24 m & Span of 0.9 m \\
\hline$N$ & 10 & 14 \\
$f$ & $0.1 \mathrm{c}$ & $0.1 \mathrm{c}$ \\
$t(\mathrm{~mm})$ & 0.1 & 0.18 \\
$\alpha$ & $90^{\circ}$ & $90^{\circ}$ \\
$K_{x}(\mathrm{~N} / \mathrm{m})$ & 301 & 1065 \\
$K_{z}(\mathrm{~N} / \mathrm{m})$ & 28 & 7.3 \\
\hline
\end{tabular}

The results with XFOIL are more accurate than those obtained with VLM. The influence of the maximum displacement limits the trend of the optimizer to maximize the number of corrugation. Table 4 illustrates that the optimizer prefers to have fewer corrugations rather than increasing the sheet thickness. This implies that the thickness has a higher impact than the number of corrugations on the axial stiffness. It can be also deduced from Table 4, that the optimizer maximizes the corrugation height and angle as they seem to have less influence on the maximum displacement.

\section{Conclusions}

The PMCM tests results show that the parametric modeling and calculation module is functioning correctly. The parametric sensitivity results show that the axial stiffness decreases as the number of corrugations, $N$, increases, as the trapezoidal corrugation angle, $\alpha$, increases, and as the half height of the corrugation, $f$, increases. The aerodynamic study showed that the maximum deflection using XFOIL is smaller than that obtained using the Tornado VLM method, and hence Tornado will give more conservative results in the optimization. XFOIL calculates aerodynamic pressures on both the upper and the lower surface of the skin, which is closer to the actual load status, and hence the deformations predicted are likely to be more accurate than those obtained with VLM. The constraint on the maximum out-of-plane displacement does force the optimizer to increase the bending stiffness of the skin for large spans. The optimum method to increase this stiffness is by reducing the number of corrugations rather than increasing the sheet thickness, since this thickness has a large impact on the axial stiffness. The optimal design also maximizes the corrugation height and the corrugation angle, which has less influence on the out-of-plane displacement.

\section{Acknowledgments}

The research leading to these results has received funding from the European Research Council under the European Union's Seventh Framework Programme (FP/2007-2013) / ERC Grant Agreement n. [247045].

\section{References}

${ }^{1}$ McCormik, B.W. Aerodynamics, Aeronautics and Flight Mechanics, 2nd Edition, Wiley, New York, 1995.

${ }^{2}$ Weisshaar, T.A. "Morphing Aircraft Technology - New Shapes for Aircraft Design," RTO-MP-AVT-141, Neuilly-sur-Seine, France, 2006.

${ }^{3}$ Thill, C., Etches, J. A., Bond, I. P., Potter, K. D. and Weaver, P. M., "Morphing Skins - A Review," The Aeronautical Journal, Vol. 112, pp. 117-139, 2008.

${ }^{4}$ Smith, D. D., Isikveren, A. T., Ajaj, R. M. and Friswell, M. I., "Multidisciplinary design optimization of an active nonplanar polymorphing wing". 27th Congress of the International Council of the Aeronautical Sciences. Nice, France, 19-24 September 2010.

${ }^{5}$ Whitehead, D.S., Kodz, M., and Hield, P.M., Reduction of Drag by Corrugating Trailing Edges. Cambridge University, Engineering Department, 1982.

${ }^{6}$ Thill, C., Downsborough, J.D., Lai, S.J., et al., "Aerodynamic study of corrugated skins for morphing wing applications", Aeronautical Journal, Vol.114, No.1154, 2010. pp. 237-244.

${ }^{7}$ Xia, Y., Bilgen, O. and Friswell, M.I., "The Effect of Corrugated Skins on Aerodynamic Performance”, ICAST2012: 23rd International Conference on Adaptive Structures and Technologies, Nanjing, China, October 11-13, 2012

${ }^{8}$ Chipperfield, A. J. and Fleming, P. J., "The Matlab Genetic Algorithm Toolbox," IEE Colloquium on Applied Control Techniques using Matlab, Digest No.1995/014, January, 1996. 
${ }^{9}$ Melin, T. A Vortex Lattice MATLAB Implementation for Linear Aerodynamic Wing Applications. Master's Thesis, Royal Institute of Technology (KTH), Department of Aeronautics, Sweden, 2000. 